\title{
Análisis y diseño de un software de gestión de procesos y costos en empresas de ensamblaje
}

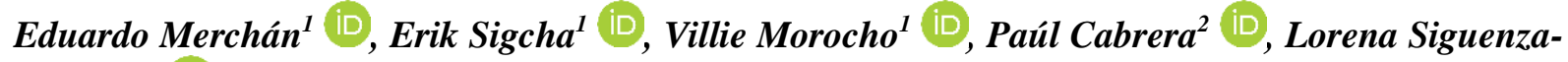 \\ Guzmán ${ }^{1}$ iD \\ ${ }^{1}$ Departamento de Ciencias de la Computación, Facultad de Ingeniería, Universidad de Cuenca, \\ Av. 12 de Abril y Agustín Cueva, Cuenca, Ecuador, 010201. \\ ${ }^{2}$ KPC Soluciones, Cuenca, Ecuador.
}

Autor para correspondencia: lorena.siguenza@ucuenca.edu.ec

Fecha de recepción: 6 de noviembre de 2017 - Fecha de aceptación: 7 de febrero de 2018

\section{RESUMEN}

En un entorno de alta competitividad empresarial, la gestión estratégica de procesos es un factor primordial para el normal desenvolvimiento de las industrias debido a su impacto directo en los objetivos de toda empresa; entre los primordiales, minimizar costos y maximizar utilidades. Desafortunadamente, su consecución se ve afectada diariamente por factores internos y externos, que inciden en el cumplimiento de los procesos realizados. El presente artículo tiene dos propósitos. Primero, documentar el análisis y diseño de una plataforma informática para el apoyo a la gestión de costos y procesos de ensamblaje, mediante el sistema de costes basados en el tiempo invertido por actividad (TDABC). Segundo, verificar si el desarrollo de la plataforma permite superar algunas de las limitaciones del sistema de costeo. Para esto, se establece primero un marco teórico que se centra en describir los principales sistemas de costeo existentes. Seguidamente, el estudio documenta el análisis y diseño de una plataforma informática haciendo uso de metodologías ágiles. El desarrollo supone una evolución de la experiencia previa de desarrollo de un prototipo que aplica TDABC para el manejo de procesos en bibliotecas. Adicionalmente, la sección de discusión presenta una interpretación sobre las principales implicaciones de usar el sistema informático como apoyo en la gestión de costos y procesos de ensamblaje. El artículo finaliza extrayendo las conclusiones más importantes del análisis efectuado.

Palabras clave: Gestión, procesos, costos, TDABC, BPMN.

\begin{abstract}
The management of strategic processes is primordial to ensure the development of any industry and is especially important given the competitive climate that every business faces while they aim to minimize costs and maximize profits. Unfortunately, the success of it is constantly impacted by internal and external factors, which affects the ability of these processes. The research pursued two primary objectives, it is to advance the knowledge of strategic and cost management processes. First, the article presents the analysis and design of a software that supports cost management and the assembly of processes using the Time Driven Activity Based Costing (TDABC). Secondly, it is argued if the development of the platform would permit to overcome some of the limitations that hindered TDABC over the years. To reach both objectives, the article provides first a theoretical framework that describes the main existing costing systems. Next, the analysis and design of an application using agile methodologies is discussed. The development of the software demonstrates an evolution coming from past experiences and lessons learned with a TDABC prototype that was used for the management of strategic processes in libraries. Further, the discussion section presents an interpretation on the main implications of using the information system as support in cost management and the assembly of processes. The article concludes by summarizing the most important conclusions from the conducted analysis.
\end{abstract}

Keywords: Management, processes, costs, TDABC, BPMN. 


\section{INTRODUCCIÓN}

El alto nivel de competitividad que se da en el entorno empresarial ha motivado a las empresas a que, en su afán por conseguir los objetivos de minimizar costos y maximizar ganancias, enfoquen su atención en la búsqueda de nuevas alternativas para la mejora de sus servicios y procesos; implicando, entre otras cosas, una buena implementación de sistemas de contabilidad de costos. En efecto, la contabilidad se ha establecido como un medio imprescindible para que las empresas conozcan de su situación económica y financiera en un determinado punto del tiempo. La contabilidad de costos, según León Mesías (2006), ha evolucionado en tres generaciones: determinación de costos unitarios, predeterminación de costos, y control de costos; surgiendo en cada una de ellas distintos sistemas de costeo. En la tercera generación, específicamente, nace el sistema de costos basados en actividades - $\mathrm{ABC}^{1}$, en el cual, además de su superioridad respecto a sus predecesores, se considera la primera aplicación de la investigación de operaciones y matemáticas en sus sistemas de cálculo (Kaplan \& Cooper, 1998). Este enfoque, para el análisis de costos, resulta importante ya que considera al factor humano como un aspecto cualitativo que acompaña al análisis cuantitativo, frecuentemente olvidado dentro de los análisis de costos, de impacto directo en sus precio y resultados (Cooper \& Kaplan, 1988).

ABC es un sistema de costos promovido por Robert S. Kaplan y Robin Cooper en 1998. Comparado con el método de costeo tradicional, $\mathrm{ABC}$ desarrolla un tratamiento más preciso y eficiente de los costos indirectos (Ellis-Newman \& Robinson, 1998). ABC primero acumula los gastos generales por cada actividad, y luego asigna los costos de las actividades a los servicios que generan esta actividad (Siguenza-Guzman, Van den Abbeele, Vandewalle, Verhaaren, \& Cattryse, 2013). Posterior a este revolucionario sistema de costeo, surge un nuevo enfoque basado en el tiempo invertido por actividad, denominado TDABC ${ }^{2}$. TDABC es un sistema de costeo desarrollado en 2004 para superar las limitaciones de sus antecesores (Kaplan \& Anderson, 2004). Este sistema recoge adecuadamente la diversidad de procesos y aporta información precisa sobre los costos (Kaplan \& Anderson, 2004). TDABC usa dos parámetros: el coste por unidad de tiempo de recurso utilizado y el tiempo estimado necesario para realizar una actividad. En la literatura sobre TDABC (Siguenza-Guzman et al., 2013; Dejnega, 2011) se describen las siguientes ventajas: 1) la facilidad y rapidez en la construcción de modelos de costos precisos; 2) la posibilidad de diseñar modelos de costos para operaciones complejas gracias a la utilización de varios controladores de tiempo; 3) la buena estimación del consumo y capacidad de los recursos; 4) la versatilidad y modularidad para mantener y construir modelos económicos de costeo; y 5) la posibilidad de utilizar TDABC de manera predictiva. Este nuevo sistema de análisis de costos proporciona datos precisos y acciones concretas de forma rápida, que pueden ser elaborados frecuentemente y a bajo costo, permitiendo a las organizaciones comprender de una mejor forma la rentabilidad del cliente (Everaert, Cleuren, \& Hoozée, 2012). El sistema TDABC, a través de las ecuaciones de tiempo, recoge de manera adecuada la diversidad de los procesos y, además, aporta una información precisa sobre los costes, un aspecto fundamental para que las empresas detecten las posibles ineficiencias internas, así como de oportunidades de desarrollo (Kaplan \& Anderson, 2007a; Everaert, Bruggeman, De Creus, \& Moreels, 2007). Este nuevo enfoque también simplifica a su predecesor, el sistema $\mathrm{ABC}$, ya que permite ser actualizado fácilmente cuando existen cambios en la estructura del modelo, además de incorporar estimaciones precisas de la capacidad de los recursos (Siguenza-Guzman et al., 2013).

Desafortunadamente, pocos son los estudios que documentan el desarrollo e implementación de sistemas informáticos para TDABC. Uno de ellos es el presentado por Cabrera y Ordoñez (2012) y Siguenza-Guzman, Cabrera, \& Cattrysse (2014) que describen el desarrollo de un módulo denominado TD-ABC-D, orientado a la gestión de costos en bibliotecas universitarias. La herramienta descrita en dichos artículos ha sido implementada como un módulo adicional en el software de gestión de bibliotecas $\mathrm{ABCD}^{3}$. Sin embargo, este sistema se encuentra enfocado a procesos en instituciones de servicios más que a procesos industriales. Más estudios son necesarios que analicen la arquitectura de estos sistemas, así como las implicaciones de su uso como apoyo a la gestión de procesos y costos de

\footnotetext{
${ }^{1}$ Por sus siglas en inglés, Activity-Based Costing

${ }^{2}$ Por sus siglas en inglés, Time-Driven Activity-Based Costing

${ }^{3}$ Más información sobre el software ABCD ir a http://wiki.bireme.org/es/index.php/ABCD
} 
ensamblaje. En este contexto, este artículo presenta el análisis y diseño de una arquitectura de software para la gestión de procesos, costos en empresas de ensamblaje basados en TDABC. Además, como pregunta de investigación, este trabajo plantea verificar si el sistema a desarrollar mejora las bondades y reduce las limitaciones de TDABC en empresas de ensamblaje.

\section{MATERIALES Y MÉTODOS}

El estudio fue desarrollado a través de un diseño cualitativo descriptivo y un enfoque fuertemente tecnológico. El enfoque para el trabajo se fundamentó en integrar todos los aspectos a tener en cuenta durante el ciclo de vida del software; es decir, las etapas por las que pasa un proyecto de software desde que es concebido, hasta que está listo para usarse (Palomo \& Gil, 2014), pero concentrándose específicamente en la descripción de las dos primeras fases del desarrollo de software: análisis de requerimientos y diseño de software (Rocha et al., 2011). Para el análisis de requerimientos se tuvo como caso de estudio una empresa local con tres líneas de ensamblaje. Esta parte cualitativa del estudio requirió un análisis de situaciones, costumbres y actitudes predominantes, a través de la descripción de los significados que les dan los miembros de la empresa a los procesos analizados. Para la parte descriptiva, se partió del diagnóstico de la situación actual, con el propósito de identificar los mejores modelos de gestión de procesos y costos de la organización. Mediante la determinación de estos puntos se pudo generar una mejor estructura para el desarrollo del sistema.

Para estas dos primeras etapas de desarrollo se ha considerado la metodología de desarrollo ágil SCRUM, que comprende a su vez cuatro fases: visualización, preparación, sprint y estabilización (De Mot, 2012). Dichas fases comprendieron el desarrollo de un conjunto de actividades, junto con la elaboración de sus productos resultantes (ver Fig. 1).

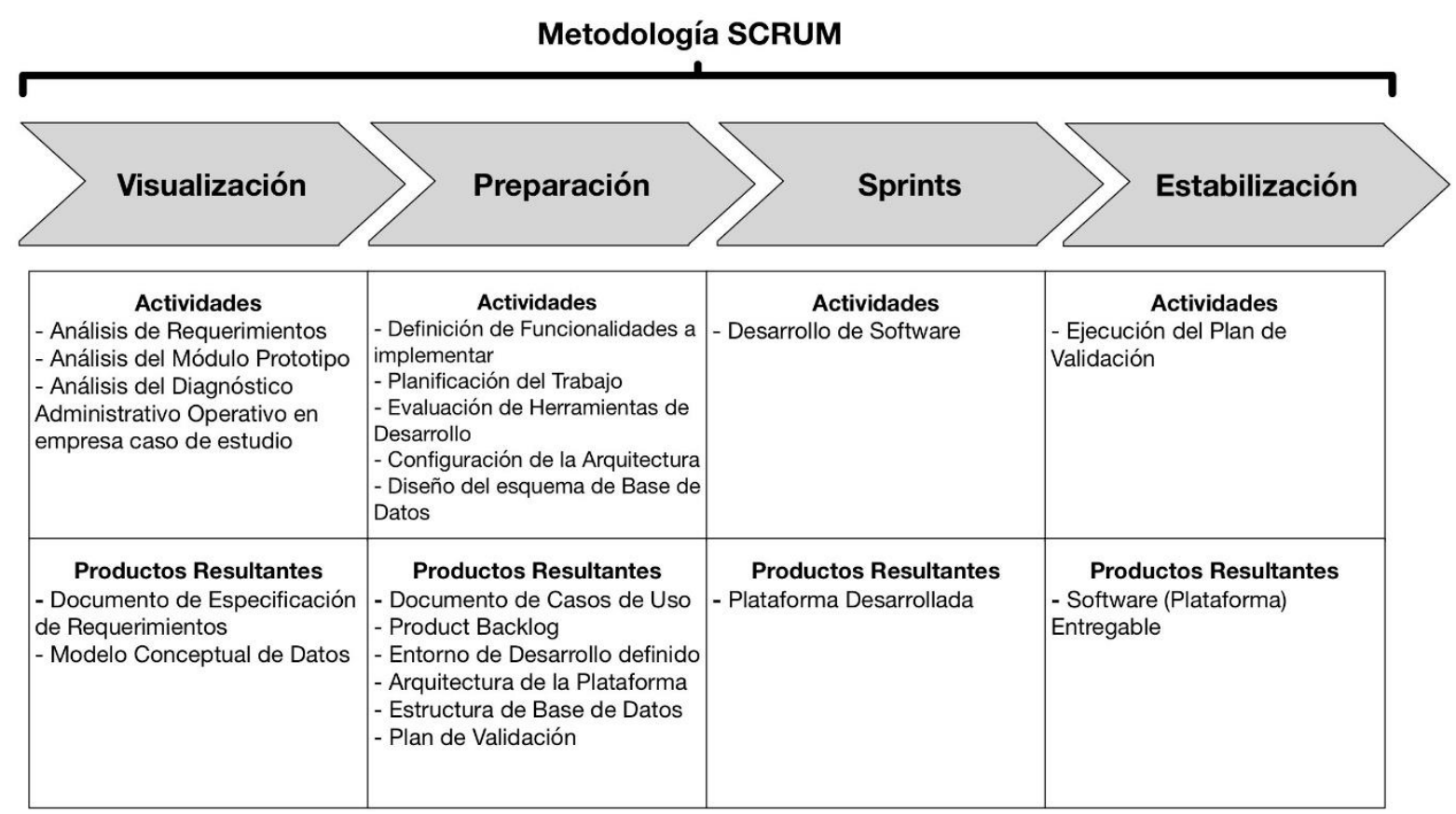

Figura 1. Metodología utilizada para el análisis y diseño del Software de Gestión de Procesos y Costos en empresas de ensamblaje aplicando SCRUM.

La fase de estabilización reúne los requerimientos del sistema, necesidades del proyecto, procesos y actores de la organización, así como riesgos e interfaces con otros sistemas. Para realizar la identificación de los requerimientos de la plataforma se partió del análisis del desarrollo del prototipo 
TD-ABC-D (Cabrera Encalada \& Ordoñez Parra, 2012) y de un diagnóstico de procesos realizado en la empresa de caso de estudio. Como resultado principal de esta fase se obtuvo un documento de "Especificación de Requerimientos", el cual contiene la prioridad de la ubicación en el sprint para priorización del desarrollo y los criterios de aceptación, los cuales serán socializados y aprobados por el cliente. Adicionalmente, se definió el modelo conceptual de datos, ilustrado en la Figura 2, el cual presenta las entidades o sujetos cuyos datos se almacenarán en la plataforma informática, así como la relación entre ellas.

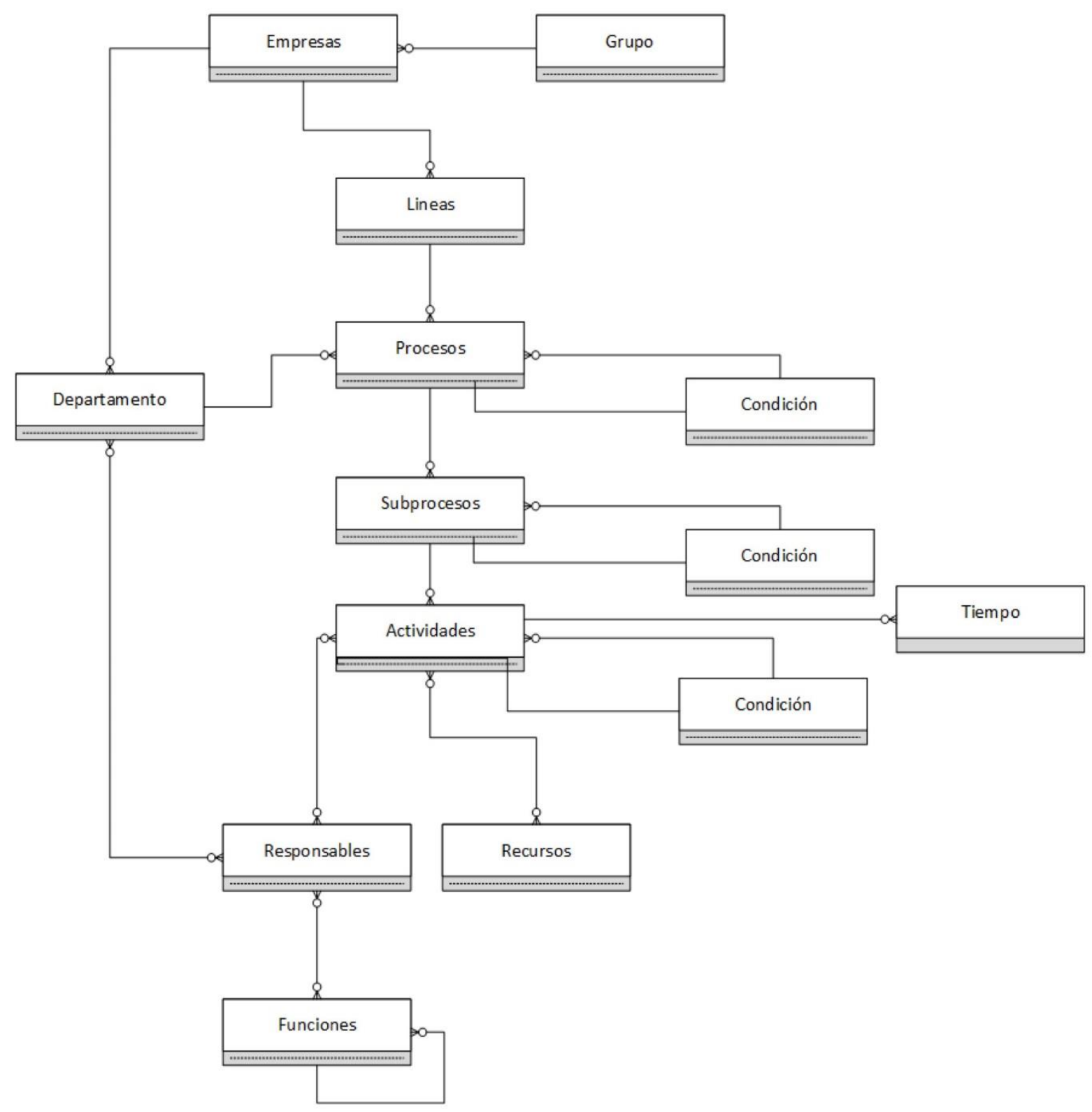

Figura 2. Modelo conceptual de datos de la plataforma.

La fase de preparación comprende la planificación del trabajo, configurar la arquitectura de la solución y listar las funcionalidades del sistema, para lo cual se analizan los requerimientos reunidos en la fase anterior. A partir de estos requerimientos se define un conjunto de funcionalidades que la plataforma debe proveer en un documento de descripción de casos de uso, el cual es utilizado para realizar la planificación del desarrollo. Con el objetivo de definir un entorno de desarrollo y una arquitectura de software, se ha realizado una evaluación de herramientas para la codificación del sistema, tomando como referencia el modelo conceptual y el listado de requerimientos funcionalidades. Se ha 
priorizado el uso de software libre y la representación de flujos de procesos aplicando la notación $\mathrm{BPMN}^{4}$ (Geiger et al., 2015). BPMN es una notación gráfica estandarizada que brinda la posibilidad de modelar procesos de negocio, describiendo la lógica de los pasos que se seguirán dentro de cada uno de estos. Dentro de la planificación, también se establece un plan de validación para la plataforma, que permita verificar el cumplimiento de los requerimientos, su correcta funcionalidad y su ajuste a la información de la empresa de caso de estudio.

La implementación del sistema se realizará en las dos últimas fases: sprint y estabilización. La fase de sprint comprende una serie de periodos de programación denominados iteraciones, los cuales se desarrollarán siguiendo la planificación definida en la fase de preparación; es decir, el uso del denominado product backlog propuesto en la metodología SCRUM. Para terminar, la fase de estabilización prueba el producto final, mediante la ejecución del "Plan de Validación de la Plataforma" y el uso de los casos de prueba establecidos en las fases anteriores. En esta fase, no se añaden nuevas funcionalidades, solo cambios pequeños que garanticen la funcionalidad correcta del software (De Mot, 2012). Como resultados de esta fase, se tienen los prototipos o entregables de cada uno de los componentes definidos en las etapas previas.

\section{RESULTADOS}

Uno de los principales resultados del estudio es la definición de una propuesta de esquema de procesos generalizado para empresas de ensamblaje en base a la cadena de valor de Porter (2001) (ver Fig. 3), el cual se ha obtenido en base al diagnóstico de procesos de las tres líneas de ensamblaje de la empresa

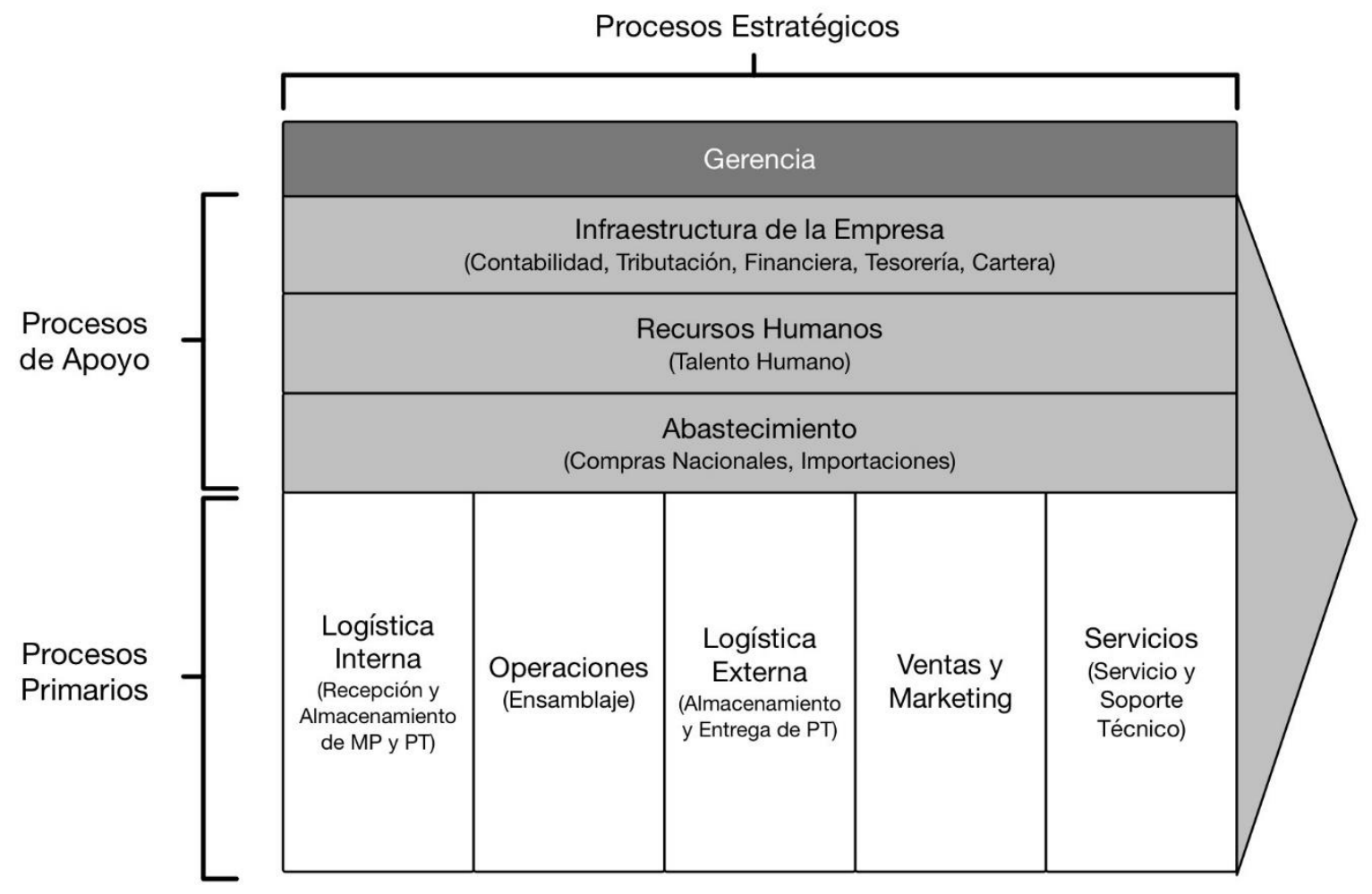

† MP: Materia Prima; PT: Productos Terminados

Figura 3. Esquema de procesos generalizado para una empresa de ensamblaje.

\footnotetext{
${ }^{4}$ Por sus siglas en inglés, Business Process Model and Notation
} 
de caso de estudio. Este esquema describe los procesos que comúnmente conforman un ciclo estándar de una línea de ensamblaje, así como los procesos que dan soporte a los demás. El esquema de procesos generalizado sirve como una referencia al momento de identificar los procesos de la empresa que se analizarán con la plataforma.

Por otro lado, la arquitectura de software resultante, basada en el patrón de arquitecturas de software Modelo-Vista-Controlador (MVC), se presenta en la Figura 4. El bloque de Fuentes de Datos representa los recursos que proporcionan datos de la empresa a la plataforma. En este bloque se destacan los valores de Tiempo por Actividad y Costo de Recurso para el cálculo TDABC, así como la metodología y sistema de calidad utilizados en cada uno de los procesos. Se incluyen también otras fuentes como archivos, sistemas transaccionales y herramientas de evaluación de calidad. El segundo bloque presenta las bases de datos y repositorios que almacenarán datos de los procesos de la empresa y los procesos de análisis de riesgo, optimización y simulación. Con estos últimos datos se podrá simular diferentes tipos de escenarios; como, por ejemplo, la variación del flujo de caja y precios de los productos en el tiempo. El bloque de Procesamiento encierra la lógica de la aplicación y controladores, los cuales permiten manipular los datos almacenados y realizar los cálculos del sistema TDABC. Finalmente, el bloque de Presentación describe la manera en que se mostrarán los datos al usuario, incluyendo los flujos de proceso y los costos obtenidos.

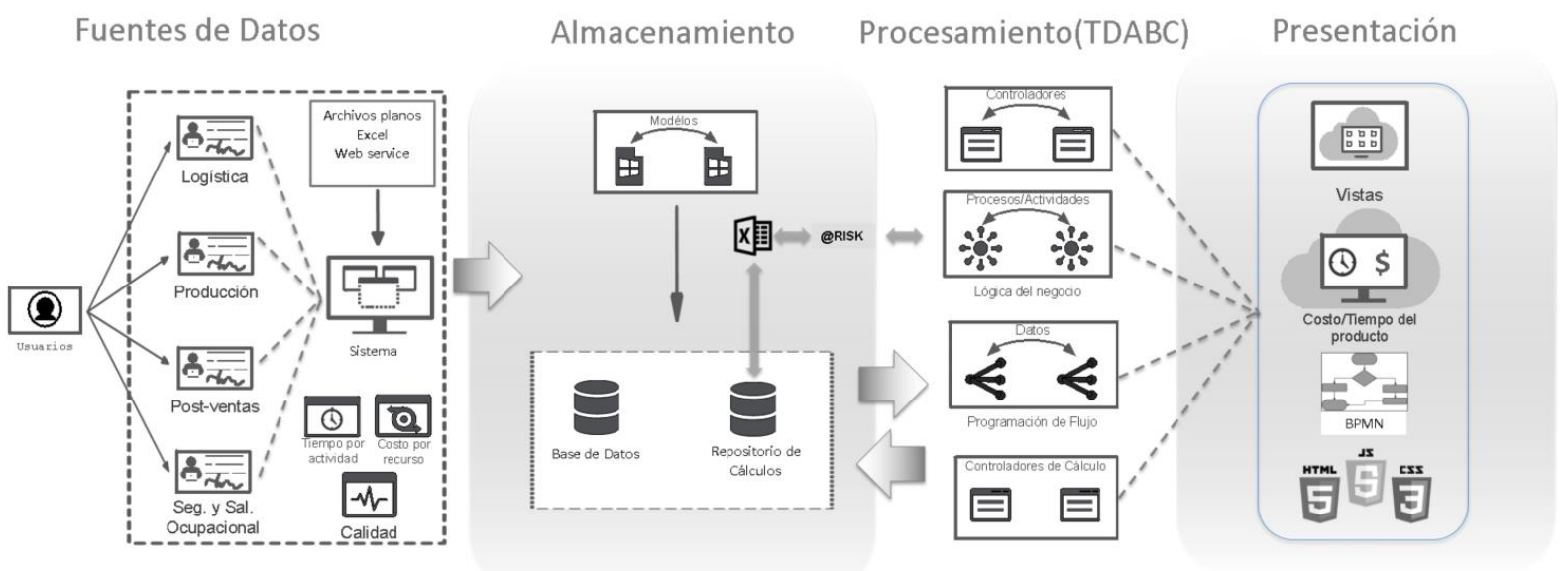

Figura 4. Arquitectura propuesta de la plataforma informática.

Otro resultado de este estudio es la planificación de la implementación y validación de la plataforma. El plan de implementación comprende un listado de las funcionalidades que ofrecerá la plataforma, junto con sus respectivos tiempos de desarrollo o sprints; estas funcionalidades serán transformadas en prototipos o entregables. Dentro del plan de validación de la plataforma, se tiene previsto evaluar el cumplimiento de las funcionalidades en base a criterios de aceptación y productos entregables definidos en los documentos de especificación de requerimientos y casos de uso. Además, se deberá validar el esquema de procesos generalizado de la Figura 3, comprobando que no solo se ajuste al diagnóstico de procesos de la empresa de caso de estudio, sino que se ajuste también a otras empresas de ensamblaje.

A más de los resultados expuestos, es posible mencionar otros productos de cada una de las fases de SCRUM. En la primera fase: 1) un documento de especificación de requerimientos de la metodología TDABC, 2) un documento con la especificación de los cálculos básicos necesarios dentro de la metodología TDABC, 3) varios documentos con las especificaciones a tomar en cuenta dentro de la metodología SCRUM para el desarrollo del product backlog. Dentro de la segunda fase se tiene: 1) la descripción de la funcionalidad de la plataforma en un documento de casos de uso, 2) el diseño de pantallas de la plataforma, 3) la planificación de la fase de implementación o product backlog, 4) la planificación de la validación de la plataforma, 5) la estructuración de los casos de prueba de cada uno de los componentes de la plataforma, y 6) la estructura de la plataforma aplicando MVC, enfocado en primer lugar en el modelado. 


\section{DISCUSIÓN}

La implementación de la plataforma de gestión de procesos y costos para empresas de ensamblaje permitirá aprovechar varias ventajas del sistema de costeo TDABC. Con el objetivo de responder la interrogante de investigación planteada en la introducción de este trabajo, a continuación, se revisan ventajas, retos, oportunidades y limitaciones del TDABC (Siguenza-Guzman et al., 2013; Namazi, 2016). Para esto se analizará cómo el uso de una herramienta de software de gestión de procesos y costos basada en TDABC podría ayudar a: 1) reducir las limitaciones planteadas por Namazi (2016) y 2) potenciar las ventajas o beneficios descritas por Siguenza-Guzman et al. (2013).

\subsection{Beneficios}

Los principales beneficios del uso de TDABC identificados por Siguenza-Guzman et al. (2013), y Namazi (2016) son: simplicidad, reducción de complejidad en operaciones, visualización de la capacidad de utilización, modularidad y versatilidad de modelos de costeo y capacidad de simulación de procesos.

En el caso de la simplicidad, TDABC simplifica la construcción de modelos de costeo, ya que solamente se requiere conocer el tiempo que toma desplegar una actividad y el costo por unidad de tiempo de desarrollo de ésta (Siguenza-Guzman et al., 2013). El uso de una herramienta de software para crear flujogramas de procesos, asignándoles tiempos y costos por actividad, hace que la construcción de modelos de costeo por procesos sea una tarea más simple e intuitiva, lo que simplifica aún más la construcción de modelos de costo. Mediante la integración con BPMN, las gráficas de flujos de procesos ayudan a los miembros de la empresa a comprender cómo se desarrollan los procesos y visualizar cuáles son las diferentes situaciones que se dan en el día a día de la empresa.

La reducción de complejidad en operaciones nos menciona que es posible el uso de las ecuaciones de tiempo, lo que permite una reducción de complejidad en los cálculos requeridos para el diseño de modelos de costos (Kaplan \& Anderson, 2007b). Esta reducción de complejidad se acentúa mediante la automatización de los cálculos de costos provista por la plataforma. El usuario del software solamente requerirá asignar responsables, tiempos y recursos utilizados a cada actividad de un proceso y el software se encargará de realizar la construcción de las ecuaciones de tiempo y calcular los resultados del análisis TDABC por procesos. De esta manera se transparenta la conformación de las ecuaciones de tiempo para cada flujo de proceso, contribuyendo a la reducción de complejidad provista por TDABC.

La visualización de la capacidad de utilización permitirá realizar varias consultas de información por departamento, proceso, subproceso o actividad, para identificar cómo se están utilizando los recursos materiales y humanos, con la finalidad de realizar una mejor planificación de su asignación para el desarrollo de las actividades. Además, se pueden obtener reportes de datos anuales por proceso en los cuales se establezca un flujo a nivel de toda la empresa para obtener un diagnóstico de esta.

La modularidad y versatilidad de modelos de costeo basados en TDABC permite la actualización de manera más sencilla que uno basado en $A B C$. Esto, ya que TDABC no requiere que se hagan entrevistas nuevamente al momento de alterar procesos (Siguenza-Guzman et al., 2013). La modularidad que provee TDABC al análisis de costos es una ventaja que se resalta aún más con la implementación de la plataforma. Esto se debe a que, la actualización de información de las actividades requerirá simplemente el ingreso de datos a la plataforma, pudiendo realizar la creación de una nueva versión de un proceso, el cual permitirá la comparación y análisis de estos. Además, en este aspecto, la plataforma TDABC puede interactuar con otros sistemas por medio del uso de servicios web o conexiones con bases de datos externas para el intercambio de información con el objetivo de mantener actualizado el modelo de costeo.

En el aspecto de la capacidad de simulación de procesos, TDABC puede usarse para modificar el comportamiento de los diferentes procesos modelados y simular las diferentes situaciones que pueden darse al cambiar valores de costos o flujos de procesos (Siguenza-Guzman et al., 2013). La plataforma aprovecha esta capacidad al momento de crear y visualizar diferentes flujos de procesos y sus versiones. Esto, sumado al uso de BPMN con su detalle de costos, permite al usuario del sistema informático identificar las posibilidades de mejora (ej. procesos, actividades, recursos y tiempo). Se puede potenciar 
este beneficio aplicando herramientas de simulación y optimización automática sobre los datos obtenidos de la plataforma mediante la generación de modelos adaptados a las necesidades específicas de la empresa.

\subsection{Limitaciones}

Dentro de las limitaciones que presenta Namazi (2016) se pueden resumir en las siguientes: el tiempo ejercido como único factor de costeo, la recopilación de datos similar al $\mathrm{ABC}$, un paso adicional en el levantamiento de datos, el no considerar la capacidad de los recursos y el comportamiento de los costos completamente y la toma de decisiones gerenciales restringida.

La aplicación de TDABC se limita a industrias donde el "tiempo" es ejercido como único factor de costeo. Esta limitación puede ser disminuida con el uso de la plataforma si se realiza el ingreso de datos de manera óptima y completa, es posible realizar un buen manejo de datos realizando cálculos desde distintos puntos de vista, logrando ejecutar comparativos de entre varios resultados, además de interactuar con sistemas externos que permitan determinar el tiempo de manera más exacta. Esto podría hacerse principalmente en los procesos en los que intervengan equipos que ayuden en la toma de tiempos. Los demás procesos que requieren una observación o encuestas todavía tendrán ese error en la medición.

La recopilación de datos es similar a un $\mathrm{ABC}$ tradicional a pesar de que da la impresión de que TDABC podría ser más sencillo. Cada vez que se actualiza y recalcula un modelo, se deben actualizar los controladores de duración; con el uso del sistema se organizará la actualización de datos y la interacción con el usuario, pero todavía no es posible la realización de una actualización de los controladores de duración de manera automática, requiriendo "mano de obra" para el cumplimiento de esta actividad; sin embargo, existe la posibilidad de importación de archivos de texto o conexiones a bases de datos de sistemas propios de la empresa como sistemas ERP5 disminuyendo un poco esta limitación.

El TDABC añade un paso más que podría considerarse innecesario para el proceso de implementación de tiempo del $\mathrm{ABC}$ porque requiere que el administrador se involucre en el proceso de la estimación del tiempo. Este proceso no sólo aumenta los costos de recolección de la información requerida, sino que también hace que consuma mucho tiempo y crea disimetría de información; al requerir la interacción humana para la estimación del tiempo, el sistema no proporcionará ayuda alguna en este sentido más que al momento del ingreso de los datos. La metodología TDABC, al calcular el costo de la capacidad no utilizada, no considera la capacidad de los recursos y el comportamiento de los costos completamente; al hacer uso del sistema es posible interactuar y hacer uso de datos de otros módulos dentro del sistema para obtener un mayor detalle en lo que se refiere a la capacidad de los recursos y el comportamiento de los costos.

La toma de decisiones con el uso de la metodología TDABC es restringida por las siguientes razones: TDABC asume que la relación entre las actividades y los recursos consumidos es lineal, absoluta y determinada, TDABC ignora las restricciones sobre los recursos de la actividad y los cuellos de botella. Principalmente, las decisiones gerenciales deben seguir el concepto de "información relevante", que no es equivalente al concepto de la información sobre costes de absorción, la información de TDABC es útil sólo cuando el tipo de decisión se define sin ambigüedad.

Mediante el uso del sistema, la inclusión de datos históricos, así como la incorporación de herramientas de optimización y análisis de datos se podría mejorar la segunda limitación en la que la recopilación de los datos es similar al $\mathrm{ABC}$ registrando los tiempos muertos de manera histórica; y en lo referente a la restricción al tomar decisiones gerenciales, al poseer resultados en base a un modelo de predicciones, las decisiones gerenciales podrían ser más exactas en base a modelos probabilísticos basados en la simulación Montecarlo. La capacidad de realizar evaluaciones comparativas de procesos es otra oportunidad que ya se aprovechó en la implementación del prototipo del módulo TDABC para bibliotecas (Cabrera Encalada \& Ordoñez Parra, 2012). Esto mediante la implementación de una funcionalidad de comparación de procesos dentro de la empresa o entre empresas del mismo grupo empresarial, la cual se ha considerado como requerimiento para la plataforma propuesta. Dentro de estos

\footnotetext{
${ }^{5}$ Por sus siglas en inglés, Enterprise Resource Planning
} 
puntos algunos que se han considerado para futuras versiones son la integración de la plataforma TDABC con una herramienta de Balance Scorecard (BSC) para proveer información de los indicadores de gestión y la integración de TDABC con sistemas de calidad, tales como Total Quality Management (TQM).

\section{CONCLUSIONES}

El análisis de costos, procesos y optimización de datos resulta importante e innovador en la industria de la producción y ensamblaje. Por este motivo, y para aprovechar las experiencias y resultados obtenidos en investigaciones previas, se ha propuesto una arquitectura para el desarrollo de un software de soporte del modelo de gestión de procesos y costos, que automatice y mejore el análisis TDABC. La implementación de una plataforma de gestión de TDABC permitirá obtener resultados de costos de forma más precisa, modelando los procesos por actividades y facilitando la visualización de los recursos sub y sobre utilizados. La plataforma ayudará a los administradores a tomar decisiones más acertadas intentando llegar al equilibrio de las capacidades de cada una de las etapas productivas.

La arquitectura presentada es el resultado de una evaluación de las herramientas y metodologías para el desarrollo de software que mejor se ajustan a los datos y funcionalidades que serán provistas por la plataforma propuesta. La arquitectura, el esquema de procesos y la plataforma resultantes serán validadas por medio de su aplicación en el análisis de los procesos de tres líneas de ensamblaje de una empresa de caso de estudio. Así también, para proceder con la implementación, se seguirán procesos iterativos y graduales que permitirán la inserción de cada uno de los componentes de forma natural. Posteriormente, se espera que esta plataforma pueda ser reutilizada por otras empresas del ámbito nacional, con la finalidad de evaluar su rendimiento y contribuir al mejoramiento de los procesos de ensamblaje.

El sistema informático brinda la posibilidad de potenciar las ventajas que posee TDABC como: simplicidad, reducción de complejidad en operaciones, visualización de la capacidad de utilización, modularidad y versatilidad de modelos de costeo y capacidad de simulación de procesos, así como también, superar algunos desafíos o limitaciones presentados por este sistema de costeo: el tiempo ejercido como único factor de costeo, la recopilación de datos similar al $\mathrm{ABC}$, un paso adicional en el levantamiento de datos, el no considerar la capacidad de los recursos y el comportamiento de los costos completamente y la toma de decisiones gerenciales restringida. Sin embargo, el diseño actual no está exento de limitaciones, que deberán ser analizadas en futuras versiones de la plataforma.

\section{AGRADECIMIENTOS}

El trabajo presentado en este artículo es parte del proyecto de investigación IMAGINE "Modelo de Gestión para la Optimización de Procesos y Costos en la Industria de Ensamblaje" ganador del XV Concurso Universitario de Proyectos de Investigación de la Dirección de Investigación de la Universidad de Cuenca. Los autores agradecen a los directivos de GO Corp. y MOTSUR por la valiosa colaboración e información brindada. Así mismo, un especial agradecimiento a todos los miembros del equipo del proyecto de investigación.

\section{REFERENCIAS}

Cabrera Encalada, P., Ordoñez Parra, C. (2012). Desarrollo de un módulo TDABC, aplicado al Centro de Documentación Regional Juan Bautista Vázquez. Tesis de Pregrado, Universidad de Cuenca, Ecuador. 
Cooper, R., Kaplan, R. S. (1988). Measure costs right: Make the right decision. Harvard Business Review, 66(5), 96-103.

De Mot, E. (2012). Agile software maintenance management. Master thesis, Faculteit Ingenieurswetenschappen, KU Leuven, Leuven, Belgium.

Ellis-Newman, J., Robinson, P. (1998). The cost of library services: Activity-based costing in an Australian academic library. Journal of Academic Librarianship, 24(5), 373-379.

Everaert, P., Cleuren, G., Hoozée, S. (2012). Using time-driven ABC to identify operational improvements: A case study in a university restaurant. Cost Management, 26(2), 41-48.

Everaert, P., Bruggeman, W., De Creus, G., Moreels, K. (2007). SANAC Logistics. Time equations to capture complexity in logistics processes (Chapter 9). In: Time-Driven Activity-Based Costing: A simpler and more powerful path to higher profits. Harvard Business Press, pp. 165-178.

Dejnega, O. (2011). Method Time Driven Activity-Based Costing - Literature review. Journal of Applied Economics, 6(Issue 1(15)), 7-15.

Geiger, M., Harrer, S., Lenhard, J., Casar, M., Vorndran, A., Wirtz, G. (2015). BPMN conformance in open source engines. IEEE Symposium on Service-Oriented System Engineering, pp. 21-30.

Kaplan, R. S., Cooper, R. (1998). Cost \& Effect: Using integrated cost systems to drive profitability and performance. Boston, MA, USA: Harvard Business School Press.

Kaplan, R. S., Anderson, S. R. (2004). Time-Driven Activity-Based Costing - Tool Kit. Harvard Business Review, 82, 131-138.

Kaplan, R. S., Anderson, S. R. (2007a). The innovation of time-driven activity-based costing. Cost Management, 21(2), 5-15.

Kaplan, R. S., Anderson, S. R. (2007b). Time-Driven Activity-Based Costing: A simpler and more powerful path to higher profits. Boston, MA, USA: Harvard Business School Press.

León Mesías, J. (2006). La comprensión de los costos en las organizaciones desde la perspectiva cualitativa. Tesis de Maestría en Ciencias de la Administración, Universidad EAFIT, 103 p. Disponible en https://repository.eafit.edu.co/bitstream/handle/10784/1168/JaimeLeon_Mesias _2006.PDF

Namazi, M. (2016). Time-driven activity-based costing: Theory, applications and limitations. Iranian Journal of Management Studies, 9(3), 457-482A.

Palomo, S. R. G., Gil, E. M. (2014). Aproximación a la ingeniería del software. Madrid, España: Editorial Universitaria Ramón Areces.

Porter, M. (2001). The value chain and competitive advantage. In: Barnes, D. (Ed.). Understanding Business: Processes. New York, NY, USA: Psychology Press, pp. 50-66.

Rocha, R. G. C., Costa, C., Rodrigues, C., Ribeiro de Azevedo, R., Junior, I. H., Meira, S., Prikladnicki, R. (2011). Collaboration models in distributed software development: a systematic review. CLEI Electronic Journal, 14(2), 1-12.

Siguenza-Guzman, L., Cabrera, P., Cattrysse, D. (2014). TD-ABC-D: Time-Driven Activity-Based Costing Software for Libraries. Presented at the 80th IFLA General Conference and Assembly, Lyon, France.

Siguenza-Guzman, L., Van den Abbeele, A., Vandewalle, J., Verhaaren, H., Cattrysse, D. (2013). Recent evolutions in costing systems: A literature review of Time-Driven Activity-Based Costing. Review of Business and Economic Literature, 58(1), 34-64. 